\title{
Hyers-Ulam Stability for Cayley Quantum Equations and Its Application to $h$-Difference Equations
}

\author{
Douglas R. Anderson and Masakazu Onitsuka®
}

\begin{abstract}
The main purpose of this study is to clarify the Hyers-Ulam stability (HUS) for the Cayley quantum equation. In addition, the result obtained for all parameters is applied to HUS of $h$-difference equations with a specific variable coefficient using a new transformation.
\end{abstract}

Mathematics Subject Classification. Primary 39A06; Secondary 39A13, 39A30, 34N05.

Keywords. Hyers-Ulam stability, Quantum equations, $q$-difference equations, $h$-difference equations.

\section{Introduction}

Quantum calculus has been of interest for some time, but really received a boost with the publication of the monograph of the same name, by Kac and Cheung [13]. In that work, both $q$-difference equations and $h$-difference equations are dealt with, but no direct transformation is given relating equations of one with the other. We introduce such a direct nexus later on in this work. First, we consider the first-order linear quantum equation

$$
D_{q} y(s)-\lambda\langle y(s)\rangle_{\gamma}=0,
$$

where $q>1, \gamma \in[0,1]$,

$$
D_{q} y(s):=\frac{y(q s)-y(s)}{(q-1) s}, \quad\langle y(s)\rangle_{\gamma}:=\gamma y(q s)+(1-\gamma) y(s),
$$

and $\lambda \in \mathbb{C}$ satisfies the condition

$$
\lambda \in \mathbb{C} \backslash\left\{\frac{-1}{(1-\gamma)(q-1) q^{k}}, \frac{1}{\gamma(q-1) q^{k}}\right\}_{k=0}^{\infty} .
$$

Equation (1.1) is called a Cayley equation, and $\gamma \in[0,1]$ is called the Cayley parameter, see [11]. Let $\mathbb{N}$ be the set of natural numbers, and let $\mathbb{N}_{0}:=\mathbb{N} \cup\{0\}$ and $q^{\mathbb{N}_{0}}:=\left\{1, q, q^{2}, q^{3}, \cdots\right\}$. 
Definition 1.1. The Cayley quantum equation (1.1) has Hyers-Ulam stability (HUS) if and only if there exists a constant $K>0$ with the following property:

For an arbitrary $\varepsilon>0$, if a function $\eta: q^{\mathbb{N}_{0}} \rightarrow \mathbb{C}$ satisfies

$$
\left|D_{q} \eta(s)-\lambda\langle\eta(s)\rangle_{\gamma}\right| \leq \varepsilon
$$

for all $s \in q^{\mathbb{N}_{0}}$, then there exists a solution $y: q^{\mathbb{N}_{0}} \rightarrow \mathbb{C}$ of $(1.1)$ such that

$$
|\eta(s)-y(s)| \leq K \varepsilon
$$

for all $s \in q^{\mathbb{N}_{0}}$.

Such a constant $K$ is called an HUS constant for (1.1) on $q^{\mathbb{N}_{0}}$.

Recently, the authors [5] considered the Hyers-Ulam stability of the Cayley quantum equation (1.1) with Cayley parameter $\gamma \in\left[0, \frac{1}{2}\right]$. Under assumption (1.2), they proved the following facts: if $\lambda=0$, then (1.1) is not HUS on $q^{\mathbb{N}_{0}}$ [5, Lemma 2.4]; if $\gamma=\frac{1}{2}$, and $\lambda \in \mathbb{C}$ satisfies (1.2), then (1.1) is not HUS on $q^{\mathbb{N}_{0}}\left[5\right.$, Theorem 3.1]; if $\gamma \in\left[0, \frac{1}{2}\right)$, and $\lambda \in \mathbb{C} \backslash\{0\}$ satisfies (1.2), then (1.1) is HUS on $q^{\mathbb{N}_{0}}[5$, Corollary 2.7]. Now arises a natural question. What happens in the case where $\gamma \in\left(\frac{1}{2}, 1\right]$ and $\lambda \in \mathbb{C} \backslash\{0\}$ satisfies (1.2)? The first purpose of this study is to consider the Hyers-Ulam stability of the Cayley quantum equation (1.1) with Cayley parameter $\gamma \in\left(\frac{1}{2}, 1\right]$. Note that the proof used here is quite different from the previous case.

In 2020, the authors [4] introduced a new, direct connection between HUS for $h$-difference equations and HUS for quantum equations of Euler type. The second purpose of this study is to establish a novel connection between HUS for Cayley quantum equations and HUS for the $h$-difference equations with a specific variable coefficient, based on the ideas in this paper.

Hyers-Ulam stability is a burgeoning area of study, encompassing functional equations, differential and difference equations, fractional equations, and the like. Some representative publications include the following. Linear $h$-difference equations and linear difference equations are explored by $[3,6,8]$, and first and second order linear equations in $[17,18]$. The Pielou logistic equation is considered by [12], and the various Möbius equations by [14-16] Implicit fractional $q$-difference equations are treated by $[1,2,10]$. Fractional stability is investigated by [7] and [20], time-dependent and periodic coefficients by [9], and differential equations and HUS driven by measures is the focus of [19].

\section{Hyers-Ulam Stability for Cayley Parameter $\gamma \in\left(\frac{1}{2}, 1\right]$}

Under the assumption (1.2), we can solve quantum equation (1.1) on $q^{\mathbb{N}_{0}}$. The following facts were given in [5]. 
Lemma 2.1. For any $\lambda$ satisfying (1.2), the general solution of (1.1) is given by

$$
y(s)=c \prod_{k=0}^{\log _{q} s-1} \frac{1+\lambda(1-\gamma)(q-1) q^{k}}{1-\lambda \gamma(q-1) q^{k}},
$$

where $c \in \mathbb{C}$ is an arbitrary constant.

Lemma 2.2. Fix $q>1$. Let $\lambda$ satisfy (1.2). For an arbitrary $\varepsilon>0$, if $a$ function $\eta: q^{\mathbb{N}_{0}} \rightarrow \mathbb{C}$ satisfies the inequality (1.3), then for $s \in q^{\mathbb{N}_{0}}$, $\eta$ has the form $\eta:=\tau \sigma+c \tau$, where

$$
\begin{aligned}
\tau(s) & :=\prod_{k=0}^{\log _{q} s-1} \frac{1+\lambda(1-\gamma)(q-1) q^{k}}{1-\lambda \gamma(q-1) q^{k}}, \\
\sigma(s) & :=\sum_{m=0}^{\log _{q} s-1} \frac{(q-1) q^{m} P\left(q^{m}\right)}{\left[1+\lambda(1-\gamma)(q-1) q^{m}\right] \tau\left(q^{m}\right)},
\end{aligned}
$$

$c \in \mathbb{C}$ is an arbitrary constant, and the function $P$ satisfies $|P(s)| \leq \varepsilon$ for all $s \in q^{\mathbb{N}_{0}}$.

Lemma 2.3. Let $q>1, \gamma \in\left(\frac{1}{2}, 1\right]$, and let $\lambda \in \mathbb{C} \backslash\{0\}$ satisfy (1.2). Let $\tau$ be the function given in (2.2). Then, $\lim _{s \rightarrow \infty}|\tau(s)|=0$, and the function

$$
|\tau(s)| \sum_{m=0}^{\log _{q} s-1}\left|\frac{(q-1) q^{m}}{\left[1+\lambda(1-\gamma)(q-1) q^{m}\right] \tau\left(q^{m}\right)}\right|
$$

is bounded above on $q^{\mathbb{N}_{0}}$.

Proof. First, we will show that $\lim _{s \rightarrow \infty} \tau(s)=0$. Since

$$
\lim _{s \rightarrow \infty}\left|\frac{1+\lambda(1-\gamma)(q-1) s}{1-\lambda \gamma(q-1) s}\right|=\frac{1}{\gamma}-1
$$

holds for $\gamma \in\left(\frac{1}{2}, 1\right]$ and $\lambda \neq 0$, we see that there exists a $s_{1} \in q^{\mathbb{N}_{0}}$ such that

$$
\left|\frac{1+\lambda(1-\gamma)(q-1) s}{1-\lambda \gamma(q-1) s}\right|<\frac{1}{2 \gamma}<1
$$

for $s \geq s_{1}$. Note here that

$$
0 \leq \frac{1}{\gamma}-1<\frac{1}{2 \gamma}<1
$$

for $\gamma \in\left(\frac{1}{2}, 1\right]$. Using the above-mentioned estimation, we have

$$
|\tau(q s)|=\left|\frac{1+\lambda(1-\gamma)(q-1) s}{1-\lambda \gamma(q-1) s}\right||\tau(s)|<\frac{1}{2 \gamma}|\tau(s)|
$$

for $s \geq s_{1}$. Let $k_{1}:=\log _{q} s_{1}$. Then,

$$
\begin{aligned}
& \left|\tau\left(q^{k_{1}+1}\right)\right|<\frac{1}{2 \gamma}\left|\tau\left(q^{k_{1}}\right)\right|, \\
& \left|\tau\left(q^{k_{1}+2}\right)\right|<\frac{1}{2 \gamma}\left|\tau\left(q^{k_{1}+1}\right)\right|<\left(\frac{1}{2 \gamma}\right)^{2}\left|\tau\left(q^{k_{1}}\right)\right|,
\end{aligned}
$$




$$
\left|\tau\left(q^{k_{1}+k}\right)\right|<\frac{1}{2 \gamma}\left|\tau\left(q^{k_{1}+k-1}\right)\right|<\cdots<\left(\frac{1}{2 \gamma}\right)^{k}\left|\tau\left(q^{k_{1}}\right)\right| .
$$

Thus, setting $s:=q^{k_{1}+k}$, we have

$$
|\tau(s)|<\left(\frac{1}{2 \gamma}\right)^{\log _{q} s-k_{1}}\left|\tau\left(q^{k_{1}}\right)\right|
$$

for $s \geq q^{k_{1}+1}=q s_{1}$. Therefore, we obtain $\lim _{s \rightarrow \infty}|\tau(s)|=0$.

Next, we will show that the function

$$
\beta(s):=|\tau(s)| \sum_{m=0}^{\log _{q} s-1}\left|\frac{(q-1) q^{m}}{\left[1+\lambda(1-\gamma)(q-1) q^{m}\right] \tau\left(q^{m}\right)}\right|
$$

is bounded on $q^{\mathbb{N}_{0}}$. First, we consider the case $\gamma \in\left(\frac{1}{2}, 1\right)$ and $\lambda \neq 0$. From

$$
\lim _{k \rightarrow \infty}\left|\frac{1+\lambda(1-\gamma)(q-1) q^{k}}{1-\lambda \gamma(q-1) q^{k}}\right|=\frac{1}{\gamma}-1
$$

we see that there exists a $k_{2} \in \mathbb{N}_{0}$ such that

$$
\left|\frac{1+\lambda(1-\gamma)(q-1) q^{k}}{1-\lambda \gamma(q-1) q^{k}}\right|<\frac{1}{2 \gamma}
$$

for $k \geq k_{2}$. Let $m \geq k_{2}$ with $m \in \mathbb{N}$. Using the same arguments as in the first part of this proof, we obtain the following:

$$
\left|\tau\left(q^{m+l}\right)\right|<\left(\frac{1}{2 \gamma}\right)^{l}\left|\tau\left(q^{m}\right)\right|
$$

for all $m \geq k_{2}$ and $l \in \mathbb{N}$. Set $s:=q^{m+l}$. Then,

$$
\left|\frac{\tau(s)}{\tau\left(q^{m}\right)}\right|<\left(\frac{1}{2 \gamma}\right)^{\log _{q} s-m}
$$

for $k_{2} \leq m \leq \log _{q} s-1$. From

$$
\lim _{m \rightarrow \infty}\left|\frac{(q-1) q^{m}}{1+\lambda(1-\gamma)(q-1) q^{m}}\right|=\lim _{m \rightarrow \infty} \frac{1}{\left|\frac{1}{(q-1) q^{m}}+\lambda(1-\gamma)\right|}=\frac{1}{(1-\gamma)|\lambda|}
$$

there exists an $m_{1} \geq k_{2}$ with $m_{1} \in \mathbb{N}_{0}$ such that

$$
\left|\frac{(q-1) q^{m}}{1+\lambda(1-\gamma)(q-1) q^{m}}\right|<\frac{2}{(1-\gamma)|\lambda|}
$$

for $m \geq m_{1}$. Consequently, we have

$$
\begin{aligned}
\beta(s) & =C_{1}|\tau(s)|+\sum_{m=m_{1}}^{\log _{q} s-1}\left|\frac{(q-1) q^{m}}{1+\lambda(1-\gamma)(q-1) q^{m}}\right|\left|\frac{\tau(s)}{\tau\left(q^{m}\right)}\right| \\
& <C_{1}|\tau(s)|+\frac{2}{(1-\gamma)|\lambda|} \sum_{m=m_{1}}^{\log _{q} s-1}\left(\frac{1}{2 \gamma}\right)^{\log _{q} s-m}
\end{aligned}
$$




$$
\begin{aligned}
& =C_{1}|\tau(s)|+\frac{2}{(1-\gamma)|\lambda|(2 \gamma)^{\log _{q} s}} \sum_{m=m_{1}}^{\log _{q} s-1}(2 \gamma)^{m} \\
& =C_{1}|\tau(s)|+\frac{2\left[1-(2 \gamma)^{m_{1}-\log _{q} s}\right]}{(2 \gamma-1)(1-\gamma)|\lambda|}
\end{aligned}
$$

for $s \geq q^{m_{1}+1}$, where

$$
C_{1}:=\sum_{m=0}^{m_{1}-1}\left|\frac{(q-1) q^{m}}{\left[1+\lambda(1-\gamma)(q-1) q^{m}\right] \tau\left(q^{m}\right)}\right| .
$$

This inequality together with $\lim _{s \rightarrow \infty}|\tau(s)|=0$ yields

$$
\lim _{s \rightarrow \infty} \beta(s) \leq \lim _{s \rightarrow \infty}\left[C_{1}|\tau(s)|+\frac{2\left[1-(2 \gamma)^{m_{1}-\log _{q} s}\right]}{(2 \gamma-1)(1-\gamma)|\lambda|}\right]=\frac{2}{(2 \gamma-1)(1-\gamma)|\lambda|} .
$$

This says that $\beta(s)$ is bounded above on $q^{\mathbb{N}_{0}}$.

Next we consider the case $\gamma=1$ and $\lambda \neq 0$. In this case, $\tau(s)$ and $\beta(s)$ are written in the following form:

$$
\tau(s)=\prod_{k=0}^{\log _{q} s-1} \frac{1}{1-\lambda(q-1) q^{k}}, \quad \beta(s)=|\tau(s)| \sum_{m=0}^{\log _{q} s-1}\left|\frac{(q-1) q^{m}}{\tau\left(q^{m}\right)}\right| .
$$

From

$$
\lim _{k \rightarrow \infty} \frac{1}{\left|\frac{1}{q^{k}}-\lambda(q-1)\right|}=\frac{1}{(q-1)|\lambda|},
$$

there exists a $k_{3} \in \mathbb{N}_{0}$ such that

$$
\frac{1}{\left|\frac{1}{q^{k}}-\lambda(q-1)\right|}<\frac{2}{(q-1)|\lambda|}
$$

for $k \geq k_{3}$, and thus,

$$
\frac{1}{\left|1-\lambda(q-1) q^{k}\right|}<\frac{2}{(q-1)|\lambda| q^{k}}
$$

for $k \geq k_{3}$. Since $\lim _{k \rightarrow \infty} \frac{2}{(q-1)|\lambda| q^{k}}=0$ holds, we see that there exists a $k_{4} \in \mathbb{N}_{0}$ such that

$$
\frac{2}{(q-1)|\lambda| q^{k}}<1
$$

for $k \geq k_{4}$. Let $m_{2}:=\max \left\{k_{3}, k_{4}\right\}$. Then,

$$
\frac{1}{\left|1-\lambda(q-1) q^{k}\right|}<\frac{1}{q^{k-m_{2}}}
$$

for $k \geq m_{2}$. Consequently, we have

$$
\begin{aligned}
& \left|\tau\left(q^{m+1}\right)\right|=\frac{\left|\tau\left(q^{m}\right)\right|}{\left|1-\lambda(q-1) q^{m}\right|}<\frac{\left|\tau\left(q^{m}\right)\right|}{q^{m-m_{2}}} \\
& \left|\tau\left(q^{m+2}\right)\right|<\frac{\left|\tau\left(q^{m+1}\right)\right|}{q^{m+1-m_{2}}}<\frac{\left|\tau\left(q^{m}\right)\right|}{q^{m+1-m_{2}} \cdot q^{m-m_{2}}} \leq \frac{\left|\tau\left(q^{m}\right)\right|}{q^{m+1-m_{2}}},
\end{aligned}
$$




$$
\left|\tau\left(q^{m+l}\right)\right|<\frac{\left|\tau\left(q^{m}\right)\right|}{q^{m+l-1-m_{2}}}
$$

for $m \geq m_{2}$ with $m \in \mathbb{N}_{0}$ and $l \in \mathbb{N}$. Put $s:=q^{m+l}$. Then,

$$
\left|\frac{\tau(s)}{\tau\left(q^{m}\right)}\right|<\frac{1}{q^{\log _{q} s-1-m_{2}}}=\frac{q^{m_{2}+1}}{s}
$$

for $m_{2} \leq m \leq \log _{q} s-1$. Using this inequality, we see that

$$
\begin{aligned}
\beta(s) & =C_{2}|\tau(s)|+\sum_{m=m_{2}}^{\log _{q} s-1}(q-1) q^{m}\left|\frac{\tau(s)}{\tau\left(q^{m}\right)}\right| \\
& <C_{2}|\tau(s)|+\frac{(q-1) q^{m_{2}+1}}{s} \sum_{m=m_{2}}^{\log _{q} s-1} q^{m} \\
& =C_{2}|\tau(s)|+q^{m_{2}+1}\left(1-\frac{q^{m_{2}}}{s}\right)
\end{aligned}
$$

for $s \geq q^{m_{2}+1}$, where

$$
C_{2}:=\sum_{m=0}^{m_{2}-1}\left|\frac{(q-1) q^{m}}{\tau\left(q^{m}\right)}\right|
$$

Thus, this together with $\lim _{s \rightarrow \infty}|\tau(s)|=0$ yields the boundedness of $\beta(s)$ on $q^{\mathbb{N}_{0}}$ when $\gamma=1$ and $\lambda \neq 0$. This completes the proof.

Theorem 2.4. Let $q>1, \gamma \in\left(\frac{1}{2}, 1\right]$, and let $\lambda \in \mathbb{C} \backslash\{0\}$ satisfy (1.2). Let $\tau$ be the function defined by (2.2). Then, (1.1) has HUS with HUS constant

$$
K:=\sup _{s \in q^{\mathbb{N}_{0}}}|\tau(s)| \sum_{m=0}^{\log _{q} s-1}\left|\frac{(q-1) q^{m}}{\left[1+\lambda(1-\gamma)(q-1) q^{m}\right] \tau\left(q^{m}\right)}\right|<\infty .
$$

on $q^{\mathbb{N}_{0}}$.

Proof. Let an arbitrary $\varepsilon>0$ be given, and $\lambda \in \mathbb{C} \backslash\{0\}$ satisfy (1.2). Assume that $\left|D_{q} \eta(s)-\lambda\langle\eta(s)\rangle_{\gamma}\right| \leq \varepsilon$ for all $s \in q^{\mathbb{N}_{0}}$. Now we consider the functions $\tau$ and $\sigma$ given in (2.2). Then, $\eta$ has the form

$$
\eta(s):=\eta_{0} \tau(s)+\tau(s) \sigma(s),
$$

and satisfies

$$
D_{q} \eta(s)-\lambda\langle\eta(s)\rangle_{\gamma}=P(s), \quad|P(s)| \leq \varepsilon
$$

for all $s \in q^{\mathbb{N}_{0}}$ by Lemma 2.2, where $\eta_{0}$ is an arbitrary complex constant. Define

$$
y(s):=\eta_{0} \tau(s) .
$$

Then, $y$ is a solution to (1.1) from Lemma 2.1. Therefore,

$$
|\eta(s)-y(s)|=|\tau(s) \sigma(s)|=|\tau(s)|\left|\sum_{m=0}^{\log _{q} s-1} \frac{(q-1) q^{m} P\left(q^{m}\right)}{\left[1+\lambda(1-\gamma)(q-1) q^{m}\right] \tau\left(q^{m}\right)}\right|
$$




$$
\leq \varepsilon|\tau(s)| \sum_{m=0}^{\log _{q} s-1}\left|\frac{(q-1) q^{m}}{\left[1+\lambda(1-\gamma)(q-1) q^{m}\right] \tau\left(q^{m}\right)}\right|
$$

for all $s \in q^{\mathbb{N}_{0}}$. By Lemma 2.3, the right-hand side is bounded above on $q^{\mathbb{N}_{0}}$. Hence, (1.1) has Hyers-Ulam stability with HUS constant

$$
K:=\sup _{s \in q^{\mathbb{N}_{0}}}|\tau(s)| \sum_{m=0}^{\log _{q} s-1}\left|\frac{(q-1) q^{m}}{\left[1+\lambda(1-\gamma)(q-1) q^{m}\right] \tau\left(q^{m}\right)}\right|<\infty .
$$

This completes the proof.

By combining Theorem 2.4 with the previous results (already mentioned in the introduction) given in [5], we get the following immediately.

Theorem 2.5. Let $q>1, \gamma \in[0,1]$, and $\lambda \in \mathbb{C}$ satisfy (1.2). Then, (1.1) has HUS on $q^{\mathbb{N}_{0}}$ if and only if $\lambda \neq 0$ and $\gamma \neq \frac{1}{2}$.

Theorem 2.6. Let $q>1$, and let $\gamma \in\left(\frac{1}{2}, 1\right]$ and $\lambda \in \mathbb{C} \backslash\{0\}$ satisfy (1.2), and

$$
\begin{aligned}
\sum_{m=0}^{\log _{q} s-1}\left|\frac{(q-1) q^{m}}{\left[1+\lambda(1-\gamma)(q-1) q^{m}\right] \tau\left(q^{m}\right)}\right| \\
\quad=\left|\sum_{m=0}^{\log _{q} s-1} \frac{(q-1) q^{m}}{\left[1+\lambda(1-\gamma)(q-1) q^{m}\right] \tau\left(q^{m}\right)}\right|
\end{aligned}
$$

for sufficiently large $s \in q^{\mathbb{N}_{0}}$. Then, (1.1) has HUS on $q^{\mathbb{N}_{0}}$. Furthermore, for sufficiently large $s \in q^{\mathbb{N}_{0}}$, there exists a $\delta>0$ such that an HUS constant is $\frac{1}{|\lambda|}+\delta$.

Proof. From the assumptions, (1.1) has HUS with HUS constant $K$ on $q^{\mathbb{N}_{0}}$, where $K$ is given in 2.3. Define $\eta_{1}(s):=-\frac{1}{\lambda}$. Then, $\eta_{1}$ is a member of the solutions to the equation

$$
D_{q} \eta(s)-\lambda\langle\eta(s)\rangle_{\gamma}=1 .
$$

On the other hand, by Lemma 2.2, we see that the general solution of this equation is written by

$$
\eta_{2}(s):=c \tau(s)+\tau(s) \sum_{m=0}^{\log _{q} s-1} \frac{(q-1) q^{m}}{\left[1+\lambda(1-\gamma)(q-1) q^{m}\right] \tau\left(q^{m}\right)},
$$

where $c \in \mathbb{C}$ is an arbitrary constant, and $\tau$ is given in (2.2). Combining these facts, we have

$$
-\frac{1}{\lambda}=c_{0} \tau(s)+\tau(s) \sum_{m=0}^{\log _{q} s-1} \frac{(q-1) q^{m}}{\left[1+\lambda(1-\gamma)(q-1) q^{m}\right] \tau\left(q^{m}\right)}
$$

for a suitable constant $c_{0} \in \mathbb{C}$. From Lemma 2.3, $\lim _{s \rightarrow \infty}|\tau(s)|=0$ holds, and so that

$$
\lim _{s \rightarrow \infty} \tau(s) \sum_{m=0}^{\log _{q} s-1} \frac{(q-1) q^{m}}{\left[1+\lambda(1-\gamma)(q-1) q^{m}\right] \tau\left(q^{m}\right)}=-\frac{1}{\lambda} .
$$


This together with the assumption in this theorem yields

$$
\lim _{s \rightarrow \infty}|\tau(s)| \sum_{m=0}^{\log _{q} s-1}\left|\frac{(q-1) q^{m}}{\left[1+\lambda(1-\gamma)(q-1) q^{m}\right] \tau\left(q^{m}\right)}\right|=\frac{1}{|\lambda|} .
$$

This means that for sufficiently large $s \in q^{\mathbb{N}_{0}}$, there exists a $\delta>0$ such that

$$
|\tau(s)| \sum_{m=0}^{\log _{q} s-1}\left|\frac{(q-1) q^{m}}{\left[1+\lambda(1-\gamma)(q-1) q^{m}\right] \tau\left(q^{m}\right)}\right| \leq \frac{1}{|\lambda|}+\delta .
$$

Therefore, $\frac{1}{|\lambda|}+\delta$ is an HUS constant for sufficiently large $s \in q^{\mathbb{N}_{0}}$.

Example 2.7. We give several examples related to Theorem 2.6. Let $\gamma=1$, $q=2$ for the following.

If $\lambda=5$, then $\delta=\frac{1}{20}$ for $s=q^{2}$, as $\left|\eta\left(2^{2}\right)-y\left(2^{2}\right)\right| \leq \frac{1}{4} \varepsilon=\left(\frac{1}{5}+\frac{1}{20}\right) \varepsilon$, and $|\eta(s)-y(s)| \leq\left|\eta\left(2^{2}\right)-y\left(2^{2}\right)\right|$ for all $s \in q^{\mathbb{N}_{0}}$.

If $\lambda=-5$, then $\delta=0$, as $|\eta(s)-y(s)| \leq \frac{1}{5} \varepsilon=\frac{1}{|-5|} \varepsilon$ for all $s \in q^{\mathbb{N}_{0}}$.

If $\lambda=1-i$, then $\delta=\frac{3}{\sqrt{5}}-\frac{1}{\sqrt{2}} \approx 0.634534$ for $s=q^{2}$, as $\left|\eta\left(2^{2}\right)-y\left(2^{2}\right)\right| \leq$ $\frac{3}{\sqrt{5}} \varepsilon=\left(\frac{1}{|1-i|}+\frac{3}{\sqrt{5}}-\frac{1}{\sqrt{2}}\right) \varepsilon$, and $|\eta(s)-y(s)| \leq\left|\eta\left(2^{2}\right)-y\left(2^{2}\right)\right|$ for all $s \in q^{\mathbb{N}_{0}}$.

Remark 2.8. Of course, condition (2.4) does not hold in general. In fact, we know that

$$
\lim _{s \rightarrow \infty}\left|\tau(s) \sum_{m=0}^{\log _{q} s-1} \frac{(q-1) q^{m}}{\left[1+\lambda(1-\gamma)(q-1) q^{m}\right] \tau\left(q^{m}\right)}\right|=\left|\frac{-1}{\lambda}\right|=\frac{1}{|\lambda|},
$$

as shown in the proof of Theorem 2.6, while numerical evidence indicates that

$$
\lim _{s \rightarrow \infty}|\tau(s)| \sum_{m=0}^{\log _{q} s-1}\left|\frac{(q-1) q^{m}}{\left[1+\lambda(1-\gamma)(q-1) q^{m}\right] \tau\left(q^{m}\right)}\right|=\frac{1}{(2 \gamma-1)|\lambda|},
$$

for any $q>1$, any $\lambda$ satisfying (1.2), and any $\gamma \in\left(\frac{1}{2}, 1\right]$. It is clear that the two limits are equal for $\gamma=1$.

\section{Application to $h$-Difference Equations}

In [4], it has been shown that there is a suitable transformation between the quantum ( $q$ and $h$ difference) equations on two different time scales to guarantee stability for both equations. More specifically, it turns out that if the $h$-difference equation has HUS, then the corresponding quantum equation of Euler type also has HUS. The reverse is also true. In this section, based on this idea, we will introduce a connection established between the Cayley quantum equation and an $h$-difference equation with variable coefficient.

Lemma 3.1. Let $q>1$ and $h>0$. Set

$$
q^{\mathbb{N}_{0}}:=\left\{1, q, q^{2}, q^{3}, \cdots\right\} \quad \text { and } \quad h \mathbb{N}_{0}:=\{0, h, 2 h, 3 h, \cdots\} .
$$


Let $\lambda \in \mathbb{C}$ satisfy (1.2), and $\alpha \in \mathbb{C}$ satisfy

$$
\alpha \in \mathbb{C} \backslash\left\{\frac{-1}{(1-\gamma) h q^{k}}, \frac{1}{\gamma h q^{k}}\right\}_{k=0}^{\infty} .
$$

Then, the Cayley quantum equation (1.1) has a solution $y$ for $s \in q^{\mathbb{N}_{0}}$ if and only if the Cayley h-difference equation

$$
q^{-\frac{t}{h}} \Delta_{h} x(t)-\alpha[x(t)]_{\gamma}=0
$$

has a solution $x$ for $t \in h \mathbb{N}_{0}$, where

$$
\Delta_{h} x(t):=\frac{x(t+h)-x(t)}{h}, \quad[x(t)]_{\gamma}:=\gamma x(t+h)+(1-\gamma) x(t),
$$

and satisfying the following relationships:

$$
t=h \log _{q} s, \quad \alpha=\frac{(q-1) \lambda}{h}, \quad \text { and } \quad x(t)=\frac{h}{q-1} y\left(q^{\frac{t}{h}}\right) .
$$

Proof. Let $y$ be a solution of (1.1) for $s \in q^{\mathbb{N}_{0}}$. From

$$
D_{q} y(s)=\frac{y\left(q^{\frac{t+h}{h}}\right)-y\left(q^{\frac{t}{h}}\right)}{(q-1) q^{\frac{t}{h}}}=q^{-\frac{t}{h}} \Delta_{h} x(t)
$$

and

$$
\langle y(s)\rangle_{\gamma}=\gamma y\left(q^{\frac{t+h}{h}}\right)+(1-\gamma) y\left(q^{\frac{t}{h}}\right)=\frac{q-1}{h}[x(t)]_{\gamma},
$$

we find that

$$
D_{q} y(s)-\lambda\langle y(s)\rangle_{\gamma}=q^{-\frac{t}{h}} \Delta_{h} x(t)-\frac{(q-1) \lambda}{h} x(t)=0 .
$$

Thus, $x$ is a solution of (3.2) for $t \in h \mathbb{N}_{0}$. The reverse is clearly true.

Remark 3.2. If $q=1+h$ and $\gamma=0$, then (3.2) is reduced to the $h$-difference equation

$$
(1+h)^{-\frac{t}{h}} \Delta_{h} x(t)-\alpha x(t)=0 .
$$

We can easily find that $(1+h)^{\frac{t}{h}}$ is a solution of $\Delta_{h} x(t)=x(t)$ for $t \in h \mathbb{N}_{0}$, and $\lim _{t \rightarrow 0}(1+h)^{\frac{t}{h}}=e^{t}$. Hence, we can regard the above $h$-difference equation as an approximate equation of the differential equation

$$
e^{-t} x^{\prime}-\alpha x=0 \text {. }
$$

Definition 3.3. The Cayley $h$-difference equation (3.2) has Hyers-Ulam stability if and only if there exists a constant $K>0$ with the following property:

For an arbitrary $\varepsilon>0$, if a function $\xi: h \mathbb{N}_{0} \rightarrow \mathbb{C}$ satisfies

$$
\left|q^{-\frac{t}{h}} \Delta_{h} \xi(t)-\alpha[\xi(t)]_{\gamma}\right| \leq \varepsilon
$$

for all $t \in h \mathbb{N}_{0}$, then there exists a solution $x: h \mathbb{N}_{0} \rightarrow \mathbb{C}$ of (3.2) such that

$$
|\xi(t)-x(t)| \leq K \varepsilon
$$

for all $t \in h \mathbb{N}_{0}$.

Such a constant $K$ is called an HUS constant for (3.2) on $h \mathbb{N}_{0}$. 
We establish the following result.

Theorem 3.4. Let $q>1, h>0, \gamma \in[0,1]$, and $\alpha \in \mathbb{C}$ satisfy (3.1). Then, (3.2) has HUS on $h \mathbb{N}_{0}$ if and only if $\alpha \neq 0$ and $\gamma \neq \frac{1}{2}$.

Proof. Suppose that $\alpha \neq 0, \gamma \neq \frac{1}{2}$, and condition (3.4) holds on $h \mathbb{N}_{0}$. Using the transformation

$$
s=q^{\frac{t}{h}}, \quad \lambda=\frac{h \alpha}{q-1}, \quad \text { and } \quad \eta(s)=\frac{q-1}{h} \xi\left(h \log _{q} s\right),
$$

we obtain

$$
q^{-\frac{t}{h}} \Delta_{h} \xi(t)=\frac{\xi\left(h \log _{q} s+h\right)-\xi\left(h \log _{q} s\right)}{h s}=D_{q} \eta(s)
$$

and

$$
[\xi(t)]_{\gamma}=\gamma \xi\left(h \log _{q} s+h\right)+(1-\gamma) \xi\left(h \log _{q} s\right)=\frac{h}{q-1}\langle\eta(s)\rangle_{\gamma} .
$$

As a result,

$$
D_{q} \eta(s)-\lambda\langle\eta(s)\rangle_{\gamma}=q^{-\frac{t}{h}} \Delta_{h} \xi(t)-\alpha[\xi(t)]_{\gamma} .
$$

This together with the assumption (3.4) says that

$$
\left|D_{q} \eta(s)-\lambda\langle\eta(s)\rangle_{\gamma}\right| \leq \varepsilon
$$

on $q^{\mathbb{N}_{0}}$. Since $\lambda=\frac{h \alpha}{q-1}, \alpha \neq 0$ and restriction (3.1) imply $\lambda \neq 0$ and condition (1.2) is met. By Theorem 2.5, we see that there exist a $K>0$ and a solution $y: q^{\mathbb{N}_{0}} \rightarrow \mathbb{C}$ of $(1.1)$ such that

$$
|\eta(s)-y(s)| \leq K \varepsilon
$$

for all $s \in q^{\mathbb{N}_{0}}$. Let $x(t)=\frac{h}{q-1} y\left(q^{\frac{t}{h}}\right)$. Then, $x$ is a solution to (3.2) by Lemma 3.1. Moreover, the above inequality implies

$$
|\xi(t)-x(t)|=\frac{h}{q-1}\left|\eta\left(q^{\frac{t}{h}}\right)-y\left(q^{\frac{t}{h}}\right)\right| \leq \frac{h K}{q-1} \varepsilon
$$

for all $t \in h \mathbb{N}_{0}$. Therefore, (3.2) has HUS on $h \mathbb{N}_{0}$ if $\alpha \neq 0$ and $\gamma \neq \frac{1}{2}$.

Conversely, we will show that HUS implies $\alpha \neq 0$ and $\gamma \neq \frac{1}{2}$. By way of contradiction, we suppose that $\alpha=0$ or $\gamma=\frac{1}{2}$ holds. Since (3.2) is HUS on $h \mathbb{N}_{0}$, we see that if (3.4) holds for $t \in h \mathbb{N}_{0}$, then there exist a $K_{0}>0$ and a solution $x: h \mathbb{N}_{0} \rightarrow \mathbb{C}$ of (3.2) such that

$$
|\xi(t)-x(t)| \leq K_{0} \varepsilon
$$

for all $t \in h \mathbb{N}_{0}$. Using transformation (3.5) again, we obtain

$$
\left|D_{q} \eta(s)-\lambda\langle\eta(s)\rangle_{\gamma}\right| \leq \varepsilon
$$

on $q^{\mathbb{N}_{0}}$. On the other hand, if $y(s)=\frac{q-1}{h} x\left(h \log _{q} s\right)$, then

$$
|\eta(s)-y(s)|=\frac{q-1}{h}\left|\xi\left(h \log _{q} s\right)-x\left(h \log _{q} s\right)\right| \leq \frac{(q-1) K_{0}}{h} \varepsilon
$$


for all $s \in q^{\mathbb{N}_{0}}$, and $y$ is a solution to (1.1) by Lemma 3.1. That is, (1.1) has HUS. However, by Theorem 2.5, we know that (1.1) is not HUS when $\alpha=0$ or $\gamma=\frac{1}{2}$. This is a contradiction.

Remark 3.5. From inequality (3.6), we conclude that the following holds: if (1.1) has HUS with HUS constant $K_{1}$ on $q^{\mathbb{N}_{0}}$, then (3.2) has HUS with HUS constant $\frac{h K_{1}}{q-1}$ on $h \mathbb{N}_{0}$. On the other hand, if (3.2) has HUS with HUS constant $K_{2}$ on $h \mathbb{N}_{0}$, then (1.1) has HUS with HUS constant $\frac{(q-1) K_{2}}{h}$ on $q^{\mathbb{N}_{0}}$. Note that if $q=h+1$, then both HUS constants are the same.

Hence, we can establish the following results using the change of variable connection given in (3.3).

Theorem 3.6. Let $q>1, h>0, \gamma \in\left(\frac{1}{2}, 1\right]$, and let $\alpha \in \mathbb{C} \backslash\{0\}$ satisfy (3.1). Then, (3.2) has HUS with HUS constant

$$
K:=\sup _{t \in h \mathbb{N}_{0}} h|\omega(t)| \sum_{m=0}^{\frac{t}{h}-1}\left|\frac{q^{m}}{\left[1+\alpha(1-\gamma) h q^{m}\right] \omega(h m)}\right|<\infty .
$$

on $h \mathbb{N}_{0}$, where

$$
\omega(t):=\prod_{k=0}^{\frac{t}{h}-1} \frac{1+\alpha(1-\gamma) h q^{k}}{1-\alpha \gamma h q^{k}}
$$

Theorem 3.7. Let $q>1, h>0$, and let $\gamma \in\left(\frac{1}{2}, 1\right]$ and $\alpha \in \mathbb{C} \backslash\{0\}$ satisfy (3.1), and

$$
\sum_{m=0}^{\frac{t}{h}-1}\left|\frac{(q-1) q^{m}}{\left[1+\alpha(1-\gamma) h q^{m}\right] \omega(h m)}\right|=\left|\sum_{m=0}^{\frac{t}{h}-1} \frac{(q-1) q^{m}}{\left[1+\alpha(1-\gamma) h q^{m}\right] \omega(h m)}\right|
$$

for sufficiently large $t \in h \mathbb{N}_{0}$. Then, (3.2) has HUS on $h \mathbb{N}_{0}$. Furthermore, for sufficiently large $t \in h \mathbb{N}_{0}$, there exists a $\delta>0$ such that an HUS constant is $\frac{h}{q-1}\left(\frac{1}{|\lambda|}+\delta\right)$.

\section{Conclusion}

In this work, we have shown for any quantum base $q>1$, any Cayley parameter $\gamma \in[0,1]$, and for eigenvalues $\lambda \in \mathbb{C}$ that satisfy a certain restriction that rules out division by zero, that the Cayley quantum equation with constant complex coefficient $\lambda$ has Hyers-Ulam stability (HUS) on the quantum time scale $q^{\mathbb{N}_{0}}$, if and only if $\lambda \neq 0$ and $\gamma \neq \frac{1}{2}$. We have also given precise estimates for the HUS constant of stability, and scenarios where it is the best (minimal) possible. Moreover, an entirely new connection is made between this Cayley quantum equation and a corresponding $h$-difference equation with variable coefficient. The HUS of one tracks exactly the HUS of the other through a change of variables, and the HUS constants are likewise related. 


\section{Acknowledgements}

The second author was supported by JSPS KAKENHI Grant Number JP20K03668.

Open Access. This article is licensed under a Creative Commons Attribution 4.0 International License, which permits use, sharing, adaptation, distribution and reproduction in any medium or format, as long as you give appropriate credit to the original author(s) and the source, provide a link to the Creative Commons licence, and indicate if changes were made. The images or other third party material in this article are included in the article's Creative Commons licence, unless indicated otherwise in a credit line to the material. If material is not included in the article's Creative Commons licence and your intended use is not permitted by statutory regulation or exceeds the permitted use, you will need to obtain permission directly from the copyright holder. To view a copy of this licence, visit http:// creativecommons.org/licenses/by/4.0/.

Publisher's Note Springer Nature remains neutral with regard to jurisdictional claims in published maps and institutional affiliations.

\section{References}

[1] Abbas, S., Benchohra, M., Laledj, N., Zhou, Y.: Existence and Ulam stability for implicit fractional q-difference equations. Adv. Differ. Equ. 2019, 12 (2019). (Paper No. 480)

[2] Abbas, S., Benchohra, M., Samet, B., Zhou, Y.: Coupled implicit Caputo fractional q-difference systems. Adv. Differ. Equ. 2019, 19 (2019). (Paper No. 527)

[3] Anderson, D.R., Onitsuka, M.: Best constant for Hyers-Ulam stability of second-order $h$-difference equations with constant coefficients. Results Math. 74(4), 16 (2019). https://doi.org/10.1007/s00025-019-1077-9. (Paper No. 151)

[4] Anderson, D.R., Onitsuka, M.: Hyers-Ulam stability for quantum equations of Euler type. Discrete Dyn. Nat. Soc. 2020(5626481), 10 (2020). https://doi.org/ $10.1155 / 2020 / 5626481$

[5] Anderson, D.R., Onitsuka, M.: Hyers-Ulam stability for quantum equations. Aequationes Math. 95, 201-214 (2021). https://doi.org/10.1007/ s00010-020-00734-1

[6] Baias, A.R., Popa, D.: On Ulam stability of a linear difference equation in Banach spaces. Bull. Malays. Math. Sci. Soc. 43(2), 1357-1371 (2020). https:// doi.org/10.1007/s40840-019-00744-6

[7] Başcı, Y., Öğrekçi, S., Mısır, A.: On Hyers-Ulam stability for fractional differential equations including the new Caputo-Fabrizio fractional derivative. Mediterr. J. Math. 16(5), 14 (2019). https://doi.org/10.1007/ s00009-019-1407-x. (Paper No. 131)

[8] Brzdȩk, J., Wójcik, P.: On approximate solutions of some difference equations. Bull. Aust. Math. Soc. 95(3), 476-481 (2017)

[9] Buşe, C., O'Regan, D., Saierli, O.: Hyers-Ulam stability for linear differences with time dependent and periodic coefficients. Symmetry 11(4), 512 (2019). https://doi.org/10.3390/sym11040512 
[10] Butt, R.I., Abdeljawad, T., Alqudah, M.A., Rehman, M.: Ulam stability of Caputo $q$-fractional delay difference equation: $q$-fractional Gronwall inequality approach. J. Inequal. Appl. 2019, 19 (2019). (Paper No. 305)

[11] Cieśliński, J.L.: Improved $q$-exponential and $q$-trigonometric functions. Appl. Math. Lett. 24(12), 2110-2114 (2011)

[12] Jung, S.-M., Nam, Y.W.: Hyers-Ulam stability of Pielou logistic difference equation. J. Nonlinear Sci. Appl. 10(6), 3115-3122 (2017)

[13] Kac, V., Cheung, P.: Quantum calculus. Springer, New York (2001)

[14] Nam, Y.W.: Hyers-Ulam stability of hyperbolic Möbius difference equation. Filomat 32(13), 4555-4575 (2018). https://doi.org/10.2298/FIL1813555N

[15] Nam, Y.W.: Hyers-Ulam stability of elliptic Möbius difference equation. Cogent Math. Stat. 5(1), 9 (2018). (Art. ID 1492338)

[16] Nam, Y.W.: Hyers-Ulam stability of loxodromic Möbius difference equation. Appl. Math. Comput. 356, 119-136 (2019)

[17] Onitsuka, M.: Influence of the step size on Hyers-Ulam stability of first-order homogeneous linear difference equations. Int. J. Differ. Equ. 12(2), 281-302 (2017)

[18] Onitsuka, M.: Hyers-Ulam stability of second-order nonhomogeneous linear difference equations with a constant step size. J. Comput. Anal. Appl. 28(1), 152-165 (2020)

[19] Satco, B.-R.: Ulam-type stability for differential equations driven by measures. Math. Nachr. 293, 147-157 (2020). https://doi.org/10.1002/mana.201800481

[20] Wang, J., Li, X.: A uniform method to Ulam-Hyers stability for some linear fractional equations. Mediterr. J. Math. 13(2), 625-635 (2016). https://doi. org/10.1007/s00009-015-0523-5

Douglas R. Anderson

Department of Mathematics

Concordia College

Moorhead MN56562

USA

e-mail: andersod@cord.edu

Masakazu Onitsuka

Department of Applied Mathematics

Okayama University of Science

Okayama 700-0005

Japan

e-mail: onitsuka@xmath.ous.ac.jp

Received: July 7, 2020.

Accepted: May 24, 2021. 